\title{
Simulation Speed Analysis and Improvements of Modelica Models for Building Energy Simulation
}

\author{
Filip Jorissen $^{1,3}$ Michael Wetter ${ }^{2}$ Lieve Helsen ${ }^{1,3}$ \\ ${ }^{1}$ Mechanical Engineering, KU Leuven, Leuven, Belgium, \{filip.jorissen, lieve.helsen\} @kuleuven.be \\ ${ }^{2}$ Energy Technologies Area, Lawrence Berkeley National Laboratory, Berkeley, CA, USA, mwetter@ lbl . gov \\ ${ }^{3}$ EnergyVille, Waterschei, Belgium
}

\begin{abstract}
This paper presents an approach for speeding up Modelica models. Insight is provided into how Modelica models are solved and what determines the tool's computational speed. Aspects such as algebraic loops, code efficiency and integrator choice are discussed. This is illustrated using simple building simulation examples and Dymola. The generality of the work is in some cases verified using OpenModelica. Using this approach, a medium sized office building including building envelope, heating ventilation and air conditioning (HVAC) systems and control strategy can be simulated at a speed five hundred times faster than real time.
\end{abstract}

Keywords: Modelica, speed, performance, buildings

\section{Introduction}

The Modelica language allows simulations of multidisciplinary problems. Combining multiple disciplines can lead to models that quickly grow in size and complexity. Consider for instance building energy modelling where building envelope, heating, ventilation and air conditioning (HVAC) systems and controls are integrated in a single model. The building envelope's thermal response typically has relatively slow dynamics, and heat transfer can be modelled using mostly linear equations. Building HVAC systems however contain a lot of non-linearities, performance curves and performance tables and typically have faster dynamics. Building control contains less dynamic components but contains a lot of discrete variables. Simulation of these types of systems can become very time consuming, limiting the use of these models.

Current literature does not provide a lot of insight into what determines computational speed and what Modelica users and library developers can do to speed up models. Chapter 14 of (Tiller, 2001) provides some hints on ways to improve computational performance such as using equations instead of algorithms, avoiding events, pro- viding Jacobians of functions, selecting good solvers and tolerances and eliminating intermediate variables. The Dymola manual, section 5.7, suggests to limit overhead for writing results and to avoid chattering, and to use options such as inline integration and parallelization (Dassault Systèmes, 2014).

While the provided tips can be valuable, they are still high-level and often do not provide a lot of insight and consequently can be difficult to apply in practice. Also, a lot of potential for code optimization remains untouched. This paper provides insight in approaches to increase computational performance of models, specifically targeted at Modelica users and Modelica library developers.

Related research focuses on creating efficient solvers such as Quantized State System (QSS) solvers, using fast Jacobian evaluation techniques and using efficient parallelization strategies. These methods can be useful and complementary, but are outside of the scope of this work.

Firstly, some technical background about Modelica is given to allow easier interpretation of the discussion. Secondly, relatively small examples are used to demonstrate how Modelica code and models can be improved in Dymola and OpenModelica. These examples are based on the IEA-EBC Annex 60 Modelica library (Wetter et al., 2015) and are available online. Finally, the code improvements are applied to a large building model, demonstrating the potential of Modelica in conjunction with the solvers available in Dymola 2015 FD01 for whole building simulations.

\section{Technical Background}

The goal of this section is to provide the technical background required for understanding the analysis performed in this paper.

\subsection{Governing Equations}

A typical Modelica model can be mathematically expressed as an implicit system of Ordinary Differential 
Equations (ODE) of the form

$$
F(t, \dot{x}, x, u)=0,
$$

with initial conditions $x(0)=x_{0}$, where $F:[0,1] \times \mathbb{R}^{n} \times$ $\mathbb{R}^{n} \times \mathbb{R}^{m} \rightarrow \mathbb{R}^{n}$, for some $n, m \in \mathbb{N}, t$ is time, $x$ is the vector of state variables and $u$ are inputs. For simplicity we omit discrete variables in this discussion. Often the equations can be manipulated analytically such that this system of equations can be expressed as an explicit ODE of the form

$$
\dot{x}=\tilde{F}(t, x, u) \text {. }
$$

For example, if a heat capacitor with capacitance $C$ is coupled to a fixed temperature boundary condition $u$ through a thermal resistor $R$, then (2) becomes

$$
\dot{x}=\frac{(u-x)}{R C} .
$$

However, if the system of ODE is coupled to algebraic equations, as is common in building simulation, such a formulation is often not possible. In this case, the problem is defined by a system of Differential Algebraic Equations (DAE) of the form

$$
\begin{aligned}
& \dot{x}=f(t, x, y, u), \\
& 0=g(t, x, y, u),
\end{aligned}
$$

with initial conditions $x(0)=x_{0}$, where $y \in \mathbb{R}^{p}$, for some $p \in \mathbb{N}$, are algebraic variables. Under certain smoothness assumptions and by use of the Implicit Function Theorem, one can show existence of a unique solution to (4) and (5) (Polak, 1997; Coddington and Levinson, 1955). This DAE can be solved by first solving (5) for $y$ and then using $y$ to compute $\dot{x}$. For example, consider a perfectly mixed volume with thermal capacity $C$ and a pump that provides a constant pressure head $\Delta p=u_{1}$. Suppose that the pump provides water to the mixing volume with temperature $u_{2}$ and that the water mass flow rate $\dot{m}=y$ is defined by a simplified pressure drop equation describing a pipe as $\dot{m}=k \sqrt{\Delta p}$ or, equivalently, $y=k \sqrt{u_{1}}$. Equations (4) and (5) are then

$$
\begin{aligned}
& \dot{x}=\frac{\left(u_{2}-x\right) \cdot y \cdot c_{p}}{C}, \\
& 0=y-k \sqrt{u_{1}},
\end{aligned}
$$

where $c_{p}$ is the specific heat capacity of water and $k$ is a constant.

\subsection{Solution of Algebraic System}

At time $t$, equation (5) needs to be solved for the algebraic variables $y$. Note that $g(\cdot, \cdot, \cdot, \cdot)$ consists of $p$ equations $0=g_{i}(\cdot, \cdot, \cdot, \cdot)$. Ideally, these can be reformulated using computer algebra and block-lower triangularization such that $y$ can be explicitly computed.
However, such a reformulation is not always possible. In our example, the solution is still relatively easy since $\dot{m}$ can be calculated directly from $\Delta p$, which is a known input. $\Delta p$ may however be a function of an algebraic variable $\dot{m}$, for instance if a proportional controller is tracking a set-point for the mass flow rate. In this case an algebraic loop is created, with two equations needing to be solved simultaneously:

$$
\begin{aligned}
& 0=\dot{m}-k \sqrt{d p}, \\
& 0=k_{p} \cdot\left(\dot{m}-\dot{m}_{s e t}\right)-d p,
\end{aligned}
$$

where $k_{p}$ is the proportional gain of the $\mathrm{P}$ controller. Note that non-linear algebraic loops are typically more expensive to solve than linear systems of equations. Dymola will try to manipulate algebraic loops to limit the amount of work required for solving them. Information about the sizes of these (non-)linear systems before and after manipulation can be found in Dymola in the Translation tab under 'Statistics'.

\subsection{Time Integration}

For simplicity, we explain the consequences of selecting explicit versus implicit time integration algorithms based on the Euler integration algorithm. Let the index $i$ denote the current time step and consider a fixed step-size Euler integration method. The explicit Euler integration method computes

$$
x_{i+1}=x_{i}+\Delta t \dot{x}_{i}=x_{i}+\Delta t f\left(t_{i}, x_{i}, y_{i}, u_{i}\right)
$$

whereas the implicit Euler integration algorithm computes

$$
x_{i+1}=x_{i}+\Delta t \dot{x}_{i+1}=x_{i}+\Delta t f\left(t_{i+1}, x_{i+1}, y_{i+1}, u_{i+1}\right) .
$$

Hence, for the implicit Euler algorithm, if $f(\cdot, \cdot, \cdot, \cdot)$ cannot be solved symbolically for $x_{i+1}$, an iterative solution is required to obtain $x_{i+1}$. This system of equations is large if there are many state variables. Solving it typically involves the calculation of the Jacobian and requires multiple iterations before convergence is achieved. This may lead to more work per time step, but it also allows large time steps being taken. Also, implicit integrators are better suited to solve stiff ODEs.

The Radau IIa integration is an implicit Runge-Kutta method. This method is a single-step method, meaning that the solution at the current time step is only affected by information from the previous time step. Integrators such as DASSL (Petzold, 1982) and Lsodar (Petzold, 1983; Hindmarsh, 1983) are multi-step methods (Dassault Systèmes, 2014). Multi-step methods use more than one previous value of the integrator's solution to approximate the new solution. For a more detailed discussion on integrators we refer to Cellier and Kofman (2006) and Hairer and Wanner (2002). 


\subsection{Simulation Procedure}

The simulation of a Modelica model typically proceeds as follows. First, the state variables are initialized based on the initial equations and start values. Then continuous time integration starts and results are saved at intermediate time intervals. At certain points in time, time or state events may occur, which need to be handled by the integrator. The equations $f(\cdot, \cdot, \cdot, \cdot)$ and $g(\cdot, \cdot, \cdot, \cdot)$ that are solved can be found in the Dymola output file dsmodel.mof in the working directory. Output of this file can be enabled in the Translation tab. Note that no distinction between equations of $f(\cdot, \cdot, \cdot, \cdot)$ and $g(\cdot, \cdot, \cdot, \cdot)$ is made in this file. The file may contain different sections that determine when the contained code is executed, such as the Initial section, Output section, Dynamics section, Accepted section and Conditionally accepted section. A description of these sections can be found in Dassault Systèmes (2014). Using dsmodel . mof and also the C-code in dsmodel . c can be important for debugging model stability and performance.

\section{Analysis of Computational Over- head}

This section builds upon the basic simulation procedure detailed above to provide further insight into reduction in computing time using illustrative examples. All numbered examples are available at https://github.com/iea-annex60/ modelica-annex60, commit e9e247d, in the Modelica package Fluid.Examples.Performance. Presented results are based on Dymola 2015 FD01 and OpenModelica 1.9.3+dev (r25881) installed on Ubuntu 14.0464 bit running on a virtual machine (Parallels 9.0.24251) on OS X Yosemite. Since the authors are most familiar with Dymola, all analyses are performed using Dymola, unless stated otherwise. A selection of results have been verified using OpenModelica to test their generality. Models that could not be compiled by OpenModelica were not verified.

The CPU time required for performing a simulation can be approximated by

$$
t=\mathscr{O}\left(t_{\text {init }}+n_{f g} \cdot t_{f g}+n_{\text {int }} \cdot t_{\text {int }}+n_{\text {data }} \cdot t_{\text {data }}\right),
$$

where $t$ are the computation times of different steps, $n$ are the number of times these steps are evaluated, and $t_{\text {init }}$ is the time required to solve the initialization problem. The indices $f g$, int, data refer, respectively, to the evaluation of functions $f(\cdot, \cdot, \cdot, \cdot)$ and $g(\cdot, \cdot, \cdot, \cdot)$, the overhead for the integrator and the data storage.

The total computational overhead can be reduced by addressing any of these components. Knowing their values provides an important hint for where

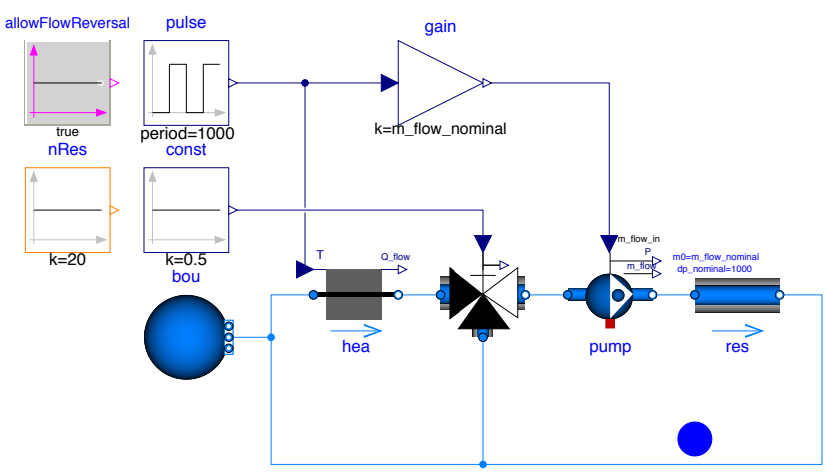

Figure 1. Example 1 illustration

computing time can be reduced. These values can be estimated from the Dymola simulation output. Setting Advanced. GenerateBlockTimers = true in Dymola generates the required output. The parameter $n_{f g}$ in (12) equals the last column of the block timers. The value of $t_{f g}$ equals the sum of column 'Mean' of rows 'OutputSection' and 'DynamicsSection'. Row 'Outside of model' contains the overhead of the integrator, and possibly other overhead as well. $n_{\text {int }}$ equals the 'Number of (succesful) steps'. $n_{\text {data }}$ is determined by the settings in the 'General' and 'Output' tabs of the simulation settings.

Decreasing any of these factors will result in a lower simulation time. However it is not always clear how this should be achieved. A measure for decreasing one factor may also cause an increase in another. The following sections provide more insight into how to influence these different factors. Firstly the overhead for each function evaluation $t_{f g}$ is discussed. Secondly the number of evaluations $n_{f g}$ is discussed. Whenever possible, example models are provided based on the Annex 60 library. Finally a methodology is proposed for increasing the simulation speed of large building models.

\subsection{Overhead per Evaluation}

Evaluation of $f(\cdot, \cdot, \cdot, \cdot)$ and $g(\cdot, \cdot, \cdot, \cdot)$ involves the evaluation of sequential code, algorithms, linear and non-linear algebraic loops, etc. We discuss how the overhead for this code can be reduced.

\subsubsection{Algebraic Loops}

When multiple equations are interdependent, an algebraic loop is formed. Depending on the type of equations the algebraic loop can be linear or non-linear. Solving non-linear algebraic loops requires iterative solutions such as encountered in a Newton-Raphson algorithm and is therefore more expensive. The user should therefore try to simplify or remove these systems where possible. We present some examples that demonstrate how this can be approached. 
Algebraic Loops Iterating on Enthalpy Consider Example 1 shown in Figure 1. The presented hydraulic system contains a heater, a three-way valve and a pump setting the mass flow rate. The pump is connected to $n R e s . k$ parallel pressure drop components res. The only two states are the temperatures of the heater and the pump with a time constant of 10 and 1 seconds, respectively. A pulsed signal sets the mass flow rate of the pump and the outlet temperature of the heater. The valve opening is set to $50 \%$. The results are generated for $n$ Res. $k=20$ unless stated otherwise.

For the given configuration Dymola generates the following algebraic loops:

Sizes nonlinear systems of equations

Sizes after manipulation

$\{6,21, \quad 46\}$

$\{1, \quad 19, \quad 22\}$

Based on the C-code generated by OpenModelica, the following algebraic loops are generated:
Sizes nonlinear systems of equations

Sizes after manipulation
$\{7, \quad 41, \quad 47\}$

$\{1, \quad 20, \quad 23\}$
In Dymola, these algebraic loops can be analysed using the dsmodel. mof file. The first system solves for the mass flow rate in the left part of the fluid loop. The second system solves for the mass flow rate in the right part of the fluid loop. The third system solves for the enthalpies of the components in the right part of the fluid loop.

Dymola's BlockTimers generate the following output for the system dynamics:

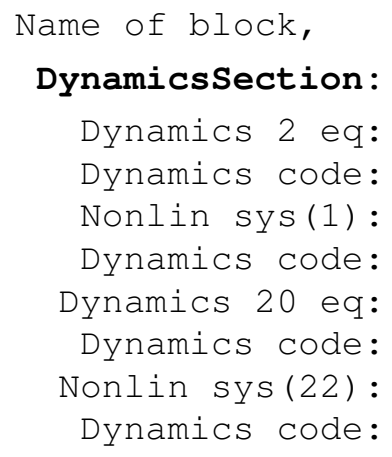

Blocks 17, 19 and 21 clearly dominate the computational cost of this example. The Dymola file dsmodel.c shows that these block numbers correspond to the three non-linear systems. We explain how these systems can be simplified or removed.

The third system is created because there are no enthalpy states in the right circuit except in the pump. In general, the fluid can flow in both directions. Therefore the inlet and outlet enthalpies of all res components can be a function of all other res components, depending on the flow direction. This causes an algebraic loop since all enthalpy values depend on each other.

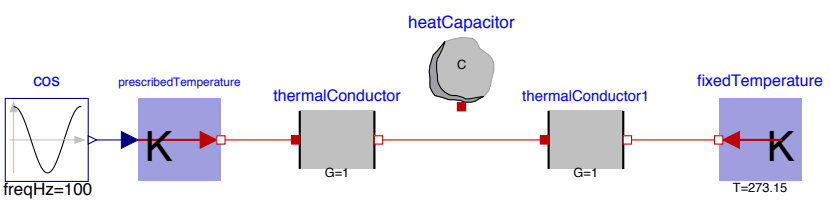

Figure 2. Example generating linear system of 2 equations

A common approach for decoupling algebraic loops is adding additional states (Zimmer, 2013). However, this can introduce fast dynamics, necessitating short time steps during parts of the simulation. The values of the state variables are solved by the integration algorithm, and hence they reduce the size of the algebraic loops. A simple example is shown in Figure 2 where a system of two linear equations is generated when the heat capacitor is unconnected. This system is decoupled when a heat capacitor is added, since the temperatures at the ports connecting the two conductances are then equal to the state variable of this heat capacitor and need no longer be obtained by solving an algebraic loop.

The enthalpy calculation of Example 1 can be simplified in a similar way by adding nRes.k mixing volumes at the location of the blue dot in Figure 1, introducing a state in the flow path with a time constant for the enthalpy of $10 \mathrm{~s}$. The state values for the enthalpy cause the system to become decoupled. The system size is now reduced from $46 / 47$ to $4 / 7$ before the manipulation, and from $22 / 23$ to $1 / 3$ after the manipulation for Dymola/OpenModelica, regardless of the value of nRes.k. Note that adding states also changes the simulation results.

In this example, a second approach is possible. We know that the fluid will always flow from the pump into the resistance. Therefore the inflow enthalpy of the resistances is always equal to the enthalpy leaving the pump. This knowledge can be passed on to the model by setting al lowFlowReversal=false in the components where no flow reversal occurs. This causes the min and max attributes of the m_flow variable of the fluid ports to be set to zero. Dymola utilizes this and simplifies equations such as

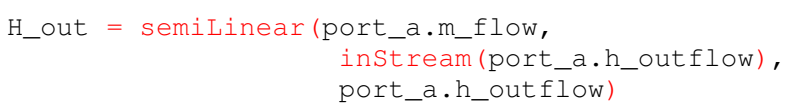

into

H_out $=$ port_a.m_flow * inStream $($ port_a.h_outflow $)$ or

H_out $=$ port_a.m_flow * port_a.h_outflow .

It can conduct this simplification because the solver can now take into account that the mass flow rate will never become negative (or positive). Due to the simplified structure of the problem, the solver is able to sort the enthalpy equations in such a way that no algebraic loop is formed: the solver can evaluate the equations sequentially, following the fluid downstream starting from 


\begin{tabular}{lcccccc}
\hline & $\begin{array}{c}\text { Succesful } \\
\text { steps }\end{array}$ & $\begin{array}{c}\text { Jacobian } \\
\text { evaluations }\end{array}$ & $\begin{array}{c}\text { Function } \\
\text { evaluations } n_{f g}\end{array}$ & $\begin{array}{c}\text { Continuous } \\
\text { time states }\end{array}$ & $\begin{array}{c}\text { Mean time } \\
\text { dynamics sec. }[\mu \text { s] }\end{array}$ & $\begin{array}{c}\text { Total time } \\
\text { dynamics sec. [s] }\end{array}$ \\
\hline N: Initial model & 55 & 21 & 647 & 2 & 310 & 0.200 \\
N: Enthalpy state & 54 & 20 & 1448 & 22 & 103 & 0.150 \\
N: No flow reversal & 55 & 21 & 647 & 2 & 109 & 0.071 \\
A: Enthalpy state & 54 & 20 & 547 & 22 & 137 & 0.075 \\
A: No flow reversal & 55 & 20 & 557 & 2 & 116 & 0.065
\end{tabular}

Table 1. Solver output for 3 configurations of Example 1 (Figure 1), with $n$ Res. $k=20$ and analytic (A) or numeric (N) Jacobian

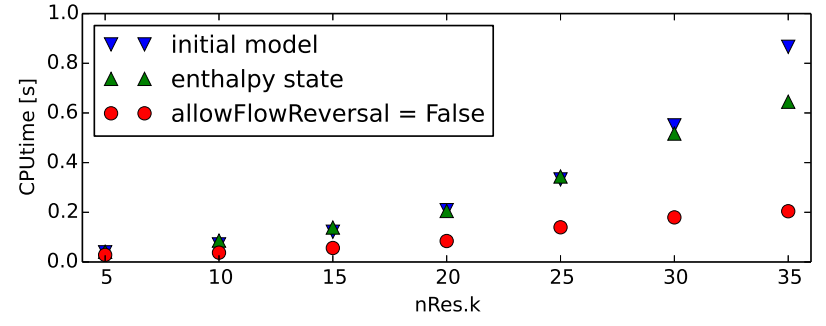

(a) numeric Jacobian

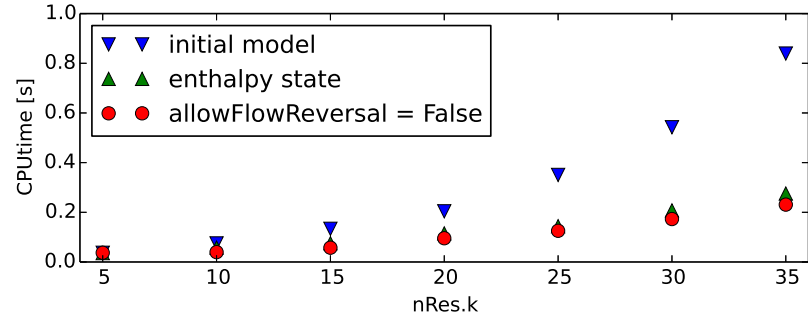

(b) analytic Jacobian

Figure 3. Simulation time for three variants of Example 1

known values of state variables. This causes the equations to be solved explicitly. OpenModelica does not make this simplification and consequently the algebraic loop size remains unchanged.

A different approach can be taken to break algebraic loops without relying on the solver to make simplifications. Many fluid components contain equations such as port_a.h_outflow = instream (port_b.h_outflow); port_b.h_outflow = instream (port_a.h_outflow);

which may be simplified into

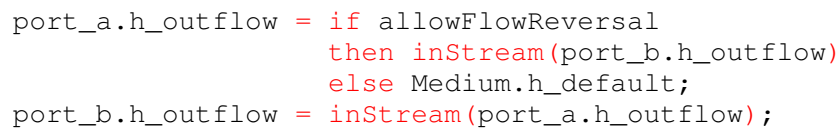

because the value of port_a.h_outflow should never be required for calculations upstream of port_a. Therefore it does not matter what its value is. Choosing a fixed value has the advantage that it allows breaking algebraic loops. Note that when the flow does reverse, the model equations will be wrong, which may cause unstable dynamics.

Figure $3 \mathrm{a}$ shows the influence of these two measures on the simulation time. Adding enthalpy states only reduced the computing time for nRes. $\mathrm{k}>20$. However, setting allowFlowReversal=false led to faster simulations. Note that the speed increase for the first case depends on the time constants of the new states. Larger time constants in general lead to faster simulations, but may introduce non-physical dynamics.

The first three rows of Table 1 allow analysing the results in further detail. Both measures allow reducing the computational work for each evaluation of $f$ and $g$ in the dynamics section from $310 \mu$ s to $\sim 106 \mu$ s. The overall speed when using allowFlowReversal= false is however better due to the lower number of function evaluations that is required: 647 instead of 1448. The increased number of function evaluations is caused by the increased number of states in the model. It turns out that the higher number of state variables leads to significantly more function evaluations, probably because by default, Dymola computes a numerical approximation to the Jacobian based on numeric differentiation.

Due to the performance penalty for approximating the Jacobian, the simulations are repeated using an analytic Jacobian, which can be done in Dymola by setting Advanced.GenerateAnalyticJacobian=true. In OpenModelica, an option for this exists in the simulation setup. Results are shown in Figure $3 \mathrm{~b}$ and in Table 1 . The penalty for adding new states is almost completely removed when using an analytic Jacobian. Somehow the average execution time for the dynamics section increased slightly, even though the equations did not change. The reason for this is unclear. The results indicate that the analytic Jacobians should be used whenever possible, especially for models with a large amount of states.

From this analysis we conclude that the user should be cautious when adding states for decoupling algebraic loops. If they are added, setting Advanced.GenerateAnalyticJacobian=true may reduce computing time. An alternative approach is to use physical insight to simplify the equations where possible, in a way similar to setting allowFlowReversal= false. Also, it may be beneficial to remove the states that are added by default in three-way valves and other components containing mixing volumes. This can be done by setting energyDynamics=massDynamics= SteadySt ate. Most likely this change will create larger systems, but often these can be simplified using the approach explained above. 


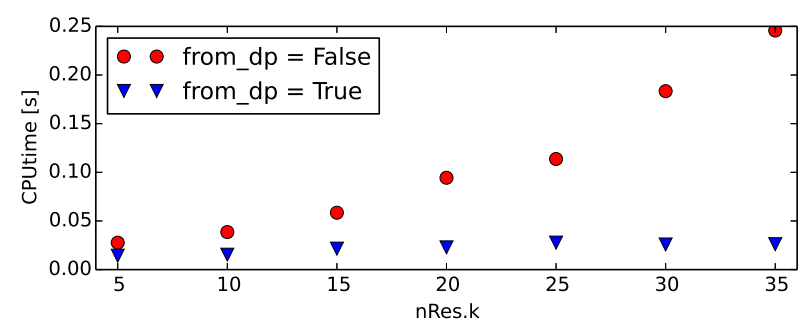

Figure 4. Example 1 illustrating computation time for solving mass flow rates through parallel resistances

\begin{abstract}
Algebraic Loops Iterating on Mass Flow Rates and Pressures When setting allowflowReversal= false, the remaining computation time is almost entirely used for computing the mass flow rates and pressures. We now focus on reducing this computing time further.
\end{abstract}

The pressure drop equations in this non-linear system can be written either as $\dot{m}=f(\Delta p)$ or as $\Delta p=f^{-1}(\dot{m})$ for some function $f(\cdot)$ or its inverse $f^{-1}(\cdot)$. The value of the parameter res.from_dp will pick one or the other formulation. If from_dp=false, then the system has size $21 / 22$ before and 19/20 after manipulation, otherwise it has sizes 21/22 and 1/1 in Dymola/OpenModelica. This can be explained as follows. When from_dp=true, the mass flow rate is calculated as a function of the pressure difference $\Delta p$. Therefore $\Delta p$ is chosen as an iteration variable. The symbolic processing algorithm detects that all resistances are in parallel and hence must have the same pressure drop. Therefore, they can all use the same iteration variable, leading to a much smaller system. This leads to a significant speed improvement, as shown in Figure 4.

Example 1 uses a pump which sets the mass flow rate to an input value and which is connected to nRes . k parallel pressure drop components. The solver can exploit the system structure by selecting the common pressure drop as an iteration variable. The "dual" problem (Example 2) could be to consider a pump which takes the pressure drop as an input value and which is connected to nRes. $k$ pressure drop components connected in series. In this case, it is advantageous to set from_dp $=f a l s e$ since Dymola and OpenModelica then select the common mass flow rate as the iteration variable, as illustrated in Figure 5.

These were fairly simple problems. In practice, combinations of parallel and series connections are used, making the choice of the parameter from_dp difficult. However, it is often possible to aggregate multiple pressure drop components that are connected in series. If all components have the same nominal mass flow rate m_flow_nominal, then the nominal pressure drops $d p$ nominal can be added into one component, reducing the series branch into a single pressure drop equation. Otherwise dp_nominal needs to be rescaled. This ap-

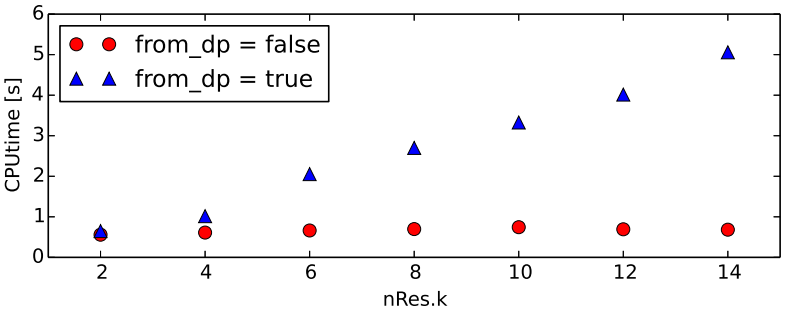

Figure 5. Example 2 illustrating computation time for solving mass flow rates through resistances in series

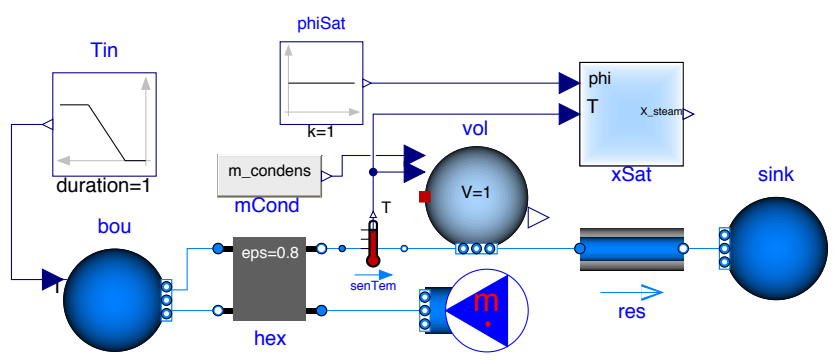

Figure 7. Example 4 illustration

proach can also be used when a valve is connected in series to the pressure drop components. The valve parameter dpFixed_nominal should then be used.

Figure 6a shows Example 3 where nRes.k parallel instances of a series connection of two resistances are simulated. The simulation time for this example is shown in Figure 6b. The parameter mergeDp indicates whether the two resistances are merged into one. Merging the two resistances gives much better results, especially when combined with from_dp=true. However when the two resistances are not merged, it is better to set from_dp=false.

Model Design for Avoiding Algebraic Loops Developers should avoid coupling systems of equations that are only weakly dependent. Consider for instance the model of a condensing heat exchanger. Such a model contains equations for the pressure drop, heat flow rate and water vapour condensation. One should try to avoid coupling these equations into one algebraic loop.

Example 4 in Figure 7 shows a simple condensing heat exchanger model. Along the flow path, first air cools in the heat exchanger hex, then condensate is extracted from the stream in vol (steady state) and finally the remaining mass is sent through a pressure drop component. Ideally the solver would be able to first compute the mass flow rate based on the pressure drop characteristic. Using this mass flow rate, the heat flow rate can be computed since it only depends on inlet temperatures and mass flow rates. Finally moisture can be extracted such that the air stream becomes saturated. In practice this sequential calculation is not possible because removing water vapour from the air affects its mass flow rate and therefore also the pressure drop. As a consequence the equations for the 


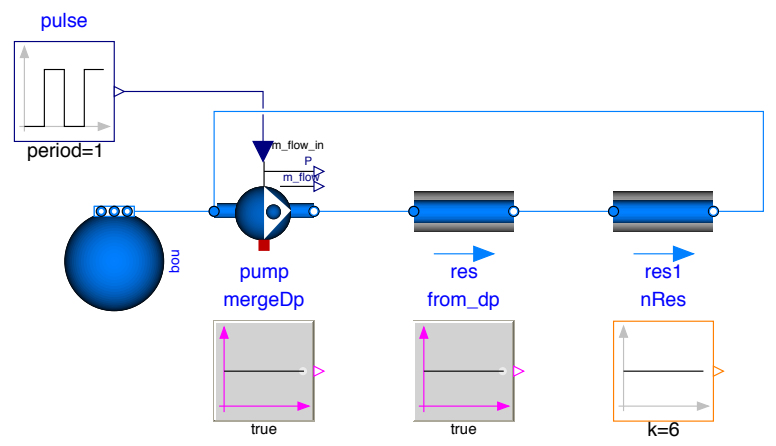

(a)

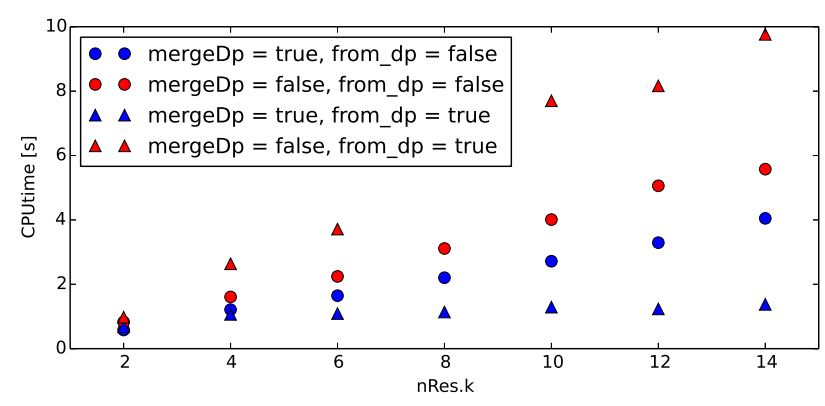

(b)

Figure 6. Example 3 (a) illustration for solving mass flow rates through parallel instancs of a series connection of two resistances and (b) simulation time based on two parameters (mergeDp and from_dp)

mass flow rate, heat flow rate and moisture balance are coupled into a single system of $12 / 10$ non-linear equations before manipulation in Dymola/OpenModelica.

As a simplification one could argue that the impact of the water vapour mass flow rate on the pressure drop is very small and that it could therefore be removed from the mass conservation equation $\sum \dot{m}=0$. This physical approximation decouples the algebraic loop so that in both simulation tools the equations can be solved sequentially.

We conclude from this discussion that the developer should consider to approximate equations if such approximations allow decoupling large systems of equations while maintaining the accuracy required by the application.

In some cases analytical solutions to nonlinear system of equations may exist. Especially linear system of equations can often be solved analytically. To enable this, the solver needs to be able to establish whether a system is linear. When using a Modelica function in a system of equations, it is therefore important that annotation (Inline=true) is used. When using this annotation, the model developer suggests to the symbolic processor to substitute the function call with the body of the Modelica function, thereby allowing the symbolic processor to detect the linearity. This allows symbolic manipulation, such that algebraic loops can be simplified.

Setting in Dymola the option Evaluate=true may also cause analytical solutions to be found, especially for linear algebraic loops. However, this leads to parameter values to be evaluated during translation, and hence they can no longer be changed without translating the model again.

These examples illustrate that even using existing component models can be a challenge. Ideally this level of complexity is not exposed to the end user. A possible approach to do this is to construct often used base circuits that are preconfigured in an efficient way.

\subsubsection{Overhead Due to Inefficient Code}

In general, every implemented equation will be evaluated. Simulation tools are able to perform certain code simplifications such as common subexpression evaluation and detection of alias variables, but the level of optimization is not exhaustive. Therefore the developer should be aware of how the solver treats equations. Here we illustrate some important aspects.

Inlining functions Inlining functions may allow better symbolic processing. It can also lower the function evaluation time, probably because overhead for calling a $\mathrm{C}$-function is avoided. We recommend to set Inline=true by default for all functions, unless their body is large.

Model Parameters Consider Example 5 shown in the code listing below:

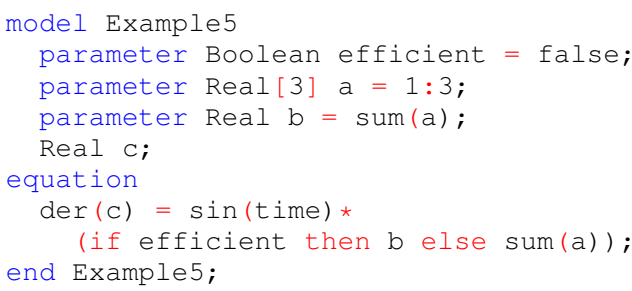

The corresponding code in dsmodel.c is

helpvar $[0]=\sin (\mathrm{Time})$;

$\mathrm{F}_{-}[0]=$ helpvar $[0] *\left(\mathrm{IF} \mathrm{DP}_{-}[0]\right.$ THEN $\mathrm{W}_{-}[0]$ ELSE $\left.\overline{D P}_{-}[1]+\mathrm{DP}_{-}[2]+\mathrm{DP}_{-}[3]\right)$;

adding annotation (Evaluate=true) to the definition of efficient results in

helpvar $[0]=\sin (\mathrm{Time})$;

$\mathrm{F}_{-}[0]=$ helpvar $[0] *\left(\mathrm{DP}_{-}[0]+\mathrm{DP}_{-}[1]+\mathrm{DP}_{-}[2]\right)$;

This can be further improved by setting efficient= true

helpvar $[0]=\sin ($ Time);

$\mathrm{F}_{-}[0]=$ helpvar $[0] * \mathrm{~W}_{-}[1]$;

The new code contains less operations, even though the implementation is mathematically identical. Taking this into account allows implementing more efficient models. 
Obsolete Model Variables In some cases it may be wise to eliminate model variables. Consider for instance variables $\mathrm{a}, \mathrm{b}$ and $\mathrm{c}$ where $b=2 a$ and $c=2 b$. If $b$ is not used in any other equation, then it is better to write $c=4 a$ and remove $b$.

It may be important to analyse the effects of such changes in detail. Consider for instance the model of a discretised wall. The model consists of a series of temperature states with an adiabatic boundary condition on one side and a sinusoidal temperature on the other side. Typically, this will be modelled using thermal capacitances $C$ and thermal resistors $R$. A Modelica implementation could be as presented by Example 6 .

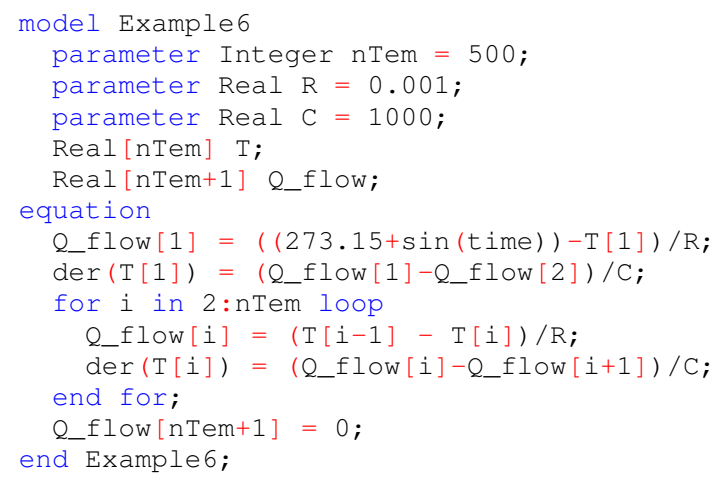

In this model variables Q_flow are calculated but not necessarily needed. These variables can be eliminated as illustrated in Example 7.

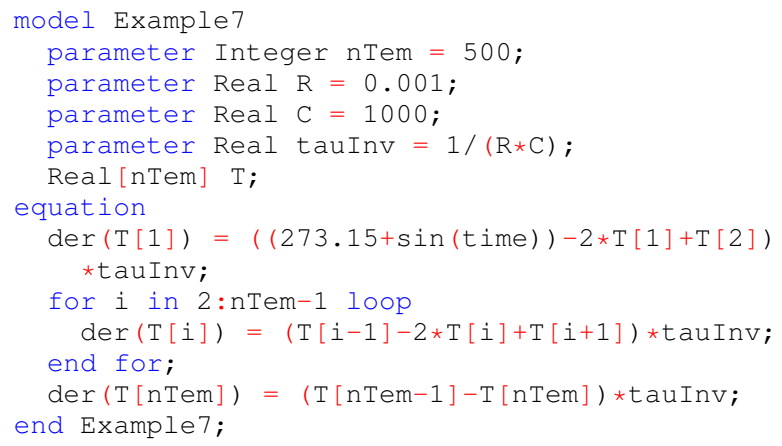

Comparing Example 7 to Example 6 a variable has been eliminated but the number of operations within the for loop remains the same. In particular, there are two additions and two divisions in Example 6, and two additions and two multiplications in Example 7. However, Example 7 is $\sim 83 \%$ faster in Dymola (2.83 s $\rightarrow 0.49 \mathrm{~s})$ and OpenModelica $(9.2 \mathrm{~s} \rightarrow 1.6 \mathrm{~s})$. It turns out that this is mostly because a division generates more overhead than a multiplication, probably because of guarding against division by zero. This performance penalty can be reduced significantly by adding annotation (Evaluate=true) to parameters $R$ and $C$, or by creating a dummy parameter similar to tau Inv and by multiplying with this parameter. This reduces simulation time to $0.65 \mathrm{~s}>0.49 \mathrm{~s}$ in Dymola and $2.39 \mathrm{~s}$ $>1.6 \mathrm{~s}$ in OpenModelica. ${ }^{1}$ The reason for the remaining

${ }^{1}$ These CPU times are based on the total Dynamics section time in Dymola and the 'simulation' timer in the Statistics output of Open- performance difference is unclear but may be explained by the extra variables $Q \_f l o w$, which may generate overhead.

From this analysis we conclude that there exists unexploited code optimization potential in popular Modelica tools. Certain variables can be eliminated and dummy parameters can be introduced to avoid parameter divisions during each time step. Until these issues are resolved, users can avoid performance penalties by taking into account these limitations by reformulating models.

Duplicate Code The developer should avoid making models that generate duplicate code. A good example is a window model, which requires the solar irradiance to be calculated. Since this calculation is influenced by parameters such as the window orientation and inclination angle, the developer may choose to include these equations in the window model. If multiple windows have the same orientation and inclination, then this means that the same calculation is repeated multiple times. This is not necessarily a problem if the overhead is small. However, in the case of a window model, the computation involves a lot of trigonometrical calculations and it would be better to isolate this calculation in a separate model. An example implementation of this problem can be found in the IDEAS library (Baetens et al., 2015). However, putting the solar irradiation in a separate model requires the user to keep the radiation computation consistent among multiple models.

An illustration of common subexpression elimination is given by Example 8 .

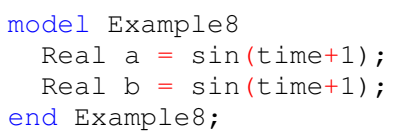

The Dymola C-code evaluates the sine and addition only once:

$W_{-}[0]=\sin (\mathrm{Time}+1) ;$ $\mathrm{W}_{-}[1]=\mathrm{W}_{-}[0]$;

This simplification is not made in OpenModelica since it evaluates the $\sin (\cdot)$ function once for $a$ and once for $b$.

Still, more complicated common subexpressions such as in IDEAS are not detected by both tools. Therefore, improving the common subexpression elimination would allow further performance improvements.

\subsection{Number of Evaluations}

The previous section focussed on how to reduce the computational overhead for each evaluation of $f(\cdot, \cdot, \cdot, \cdot)$ and $g(\cdot, \cdot, \cdot, \cdot)$. The current section focusses on how to reduce the number of evaluations. Important aspects are the time constants of the system, the system stability, the number of events, computing the Jacobian and the integrator choice.

Modelica when performing 100000 Euler integration steps of Example 6 and Example 7. 
System Time Constants When a system has fast dynamics, then the solver has to track these dynamics with small step sizes. In general, systems with large time constants have shorter calculation times. It may therefore be advantageous to make certain dynamics slower, especially the fastest dynamics in the system. Dymola option "Which states that dominate error" may be used to identify these states. Changing the dynamics may however be non-physical or introduce instability in feedback control loops. In this case a different option may be to remove the fast dynamics completely and simulate the system as a steady state system. Note, however, that this may increase the size of the algebraic system of equations.

The latter approach may be very effective when considering air flow networks. If air is modelled as compressible, pressure states are created in instances of MixingVolume, unless massDynamics= Steadystate. These states however introduce small time constants if part of a building air flow network. It may therefore be better to remove them. Again, this may create larger systems of equations.

System Stability If a feedback control loop is tuned badly, oscillatory behaviour can occur. A variable time step integrator may track these oscillations, leading to a major decrease in simulation speed. Note that it may be difficult to see these oscillations when the output interval is set too large.

Number of Events Events require the integration to stop and restart, typically with a lower order method and with smaller time steps. In addition, for state events, typical ODE solvers require an iterative solution to find the time when the event happens.

Computing the Jacobian Some integrators require the Jacobian to be calculated. Having more states leads to a larger Jacobian, as was illustrated in Example 1. Since by default, Dymola and OpenModelica use numeric differentiation to approximate the Jacobian, a lot of finite differences need to be calculated, each requiring a function evaluation. Note that in particular models with a larger number of states benefit more from having an analytic Jacobian, since the number of Jacobian entries equals the square of the number of states.

Integrator Choice Many integrators use an implicit integration scheme. This typically requires the computation of a Jacobian and requires iterations to be performed before reaching convergence. This can lead to more function evaluations. However, for stiff systems, implicit integrators are more efficient than explicit integrators.

\subsection{Analysis of Large Problems}

In the previous sections, computing time was analysed using small models. In building simulation, models can however become considerably larger and analysing the computational speed can be difficult since it depends on a lot of factors, including the unknown solver implementation. Still, we predict some trends for the computation time, based on the size of the model.

Consider a model of a district energy system, including building models and an electrical grid. When doubling the size of the district, ideally the computational time would double as well, such that computational time scales linearly with problem size. Let us analyse this further based on Equation 12. Ideally $t_{f g}$ scales linearly with the problem size. In practice this is not necessarily the case. The electrical grid of the district typically results in a large non-linear system of equations since all electrical components have very fast transients and are therefore modelled as steady state components. Doubling the size of the model therefore also doubles the size of the algebraic loop. Example 1 has shown that computational time for algebraic loops does not scale linearly with size and therefore larger models will become computationally slow. Equations outside algebraic loops can be solved sequentially. Therefore their computational time does scale linearly.

Because $t_{f g}$ scales, at best, linearly with size, $n_{f g}$ should remain constant if we want to obtain overall linear scaling of the computational time. However, firstly, generally $n_{f g}$ also grows with problem size, for example because larger problems have more controllers that may trigger events. If the amount of buildings doubles, then the amount of state events may double, which causes a performance penalty. Secondly, when a numeric Jacobian needs to be computed, then $n_{f g}$ will increase since the number of states increases linearly with the problem size. The number of operations for an implicit integrator typically does not scale linearly either. Solving dense implicit systems typically requires $\mathscr{O}\left(n^{3}\right)$ operations (Hairer and Wanner, 2002). Building model Jacobians are however very sparse. It is not clear how well this is exploited by Dymola. An integrator such as Rkfix 4 can have an operation count that is linear with the problem size, unless the fixed time step is changed. For certain large problems that do not require event handling, it can therefore be advantageous to use these simple integrators, also because they do not require a Jacobian to be calculated.

\subsubsection{Parallelization}

Dymola supports parallelization for the calculation of $f(\cdot, \cdot, \cdot, \cdot)$ and $g(\cdot, \cdot, \cdot, \cdot)$ (Dassault Systèmes, 2014) and analytic Jacobian (see Advanced.ParallelizeAnalyticJacobian). However parallelization generates overhead for syn- 


\begin{tabular}{|c|c|c|c|c|c|c|c|c|c|}
\hline Integrator & $\begin{array}{l}\text { Tolerance } \\
\text { / step size }\end{array}$ & $\begin{array}{r}\text { CPUtime } \\
{[\mathrm{s}]}\end{array}$ & $\begin{array}{l}\text { Dynamics } \\
\text { section [s] }\end{array}$ & $\begin{array}{r}\text { Outside of } \\
\text { model [s] }\end{array}$ & $\begin{array}{l}\text { Function } \\
\text { evaluations } n_{f g}\end{array}$ & $\begin{array}{r}\text { State } \\
\text { events }\end{array}$ & $\begin{array}{r}\text { Time } \\
\text { events }\end{array}$ & $\begin{array}{r}\text { Jacobian } \\
\text { evaluations }\end{array}$ & $\begin{array}{r}E_{e l} \\
\text { [error] }\end{array}$ \\
\hline Dassl & $1 \mathrm{E}-6$ & 4261 & 3538 & 476 & 787341 & 41 & 8 & 1235 & -4.35 E-6 \\
\hline Dassl & $1 \mathrm{E}-4$ & 3088 & 2759 & 327 & 546326 & 36 & 8 & 862 & 3.17 E-3 \\
\hline Radau IIa & $1 \mathrm{E}-6$ & 4042 & 2400 & 1416 & 453073 & 37 & 8 & 347 & 1.64 \\
\hline Lsodar & $1 \mathrm{E}-6$ & 3450 & 2666 & 547 & 679486 & 44 & 8 & 1047 & -4.35 E-6 \\
\hline Lsodar & $1 \mathrm{E}-4$ & 2073 & 1435 & 515 & 347018 & 41 & 8 & 537 & $-2.25 \quad E-5$ \\
\hline Lsodar & $1 \mathrm{E}-2$ & 1655 & 1152 & 406 & 256458 & 38 & 8 & 399 & 4.51 E-3 \\
\hline Dopri45 & $1 \mathrm{E}-6$ & 194 & 159 & 17.0 & 41166 & 39 & 8 & 0 & 4.68 \\
\hline Dopri45 & $1 \mathrm{E}-8$ & 199 & 162 & 18.3 & 42017 & 39 & 8 & 0 & 1.96 \\
\hline Rkfix4 & $20 \mathrm{~s}$ & 15.4 & 11.3 & 1.1 & 2717 & 39 & 8 & NA & 1.34 E-2 \\
\hline Rkfix4 & $5 \mathrm{~s}$ & 50.6 & 42.9 & 1.5 & 10233 & 43 & 8 & NA & 2.52 \\
\hline Rkfix4 & $1 \mathrm{~s}$ & 224 & 202 & 3.2 & 50211 & 38 & 8 & NA & 1.28 \\
\hline Euler & $5 \mathrm{~s}$ & 24.0 & 18.2 & 1.7 & 4271 & 50 & 8 & NA & -2.00 \\
\hline Euler & $0.25 \mathrm{~s}$ & 446 & 389 & 12.8 & 80233 & 41 & 8 & NA & -4.22 E-4 \\
\hline
\end{tabular}

Table 2. Example building model statistics for various integrators and tolerance options. Results are the solution statistics (when available, else 'NA') and the relative error of $E_{e l}$

chronization and communication. The authors have not been able to gain notable improvements in simulation speed in building applications by using parallelization in Dymola 2015 FD01.

\subsubsection{Example of Large Building Model}

The approach explained in this paper was applied to a building model based on a real case (Solarwind, Luxemburg), containing 32 IDEAS (Baetens et al., 2015) building zones with individual concrete core activation circuits (Baetens et al., 2015) and Variable Air Volume (VAV) boxes including heating battery, bore field model (Picard and Helsen, 2014), solar collector (Wetter et al., 2014), four thermal storage devices (Wetter et al., 2014), one pellet boiler, four heat pumps (Baetens et al., 2015), two adiabatic/active heat recuperating air handling units, pumps (Wetter, 2013) and valves (Wetter et al., 2015) and a control strategy based mostly on hysteresis controllers, PID controllers, heating/cooling curves and boolean algebra. The model has 2468 continuous time states and 28342 time-varying variables.

Special care was taken to make sure that the smallest time constants are in the order of $30 \mathrm{~s}$. Therefore air ducts are steady state, pumps and valves have no opening delay or filter and pipes were lumped into only a few states per circuit branch, thereby allowing to increase the time constant. Temperature sensors are assumed to have a time constant in the order of one minute. Using dynamic sensors avoids coupling the thermal equations with the control equations into a single algebraic loop.

This model was simulated for $t_{\text {end }}-t_{\text {start }}=10000 \mathrm{~s}$ using various implicit integrators, with numeric Jacobians and explicit integrator Dopri 45. The total amount of function evaluations exceeds 40000 in each case. This is on average one function evaluation every $0.25 \mathrm{~s}$, while the smallest time constant of the system is $\sim 30 \mathrm{~s}$. Therefore it makes sense to use an explicit fixed step integrator. Table 2 shows the results, including fixed step ex-

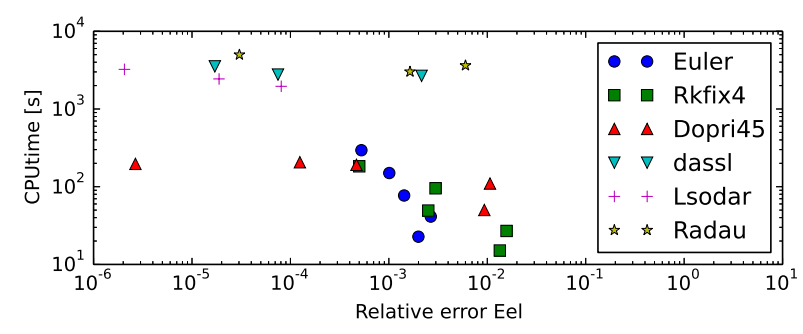

Figure 8. Relative errors of $E_{e l}$ for various solvers and tolerances or fixed time step sizes

plicit integrators Rkfix4 and Euler. It contains statistics and the error on one simulation result that is of interest, namely the integrated electrical power consumption of the building $E_{e l}$. The relative error was calculated using Dassl with a tolerance of $10^{-8}$, which produced a result of $4.591880 \mathrm{kWh}$.

From these results and Figure 8 it is clear that implicit integrators are very slow compared to explicit integrators for this problem. Fixed step methods are especially fast when high accuracy is not required, allowing a simulation speed 500 times faster than real time, which is more than 100 times faster than Dassl. For higher accuracies, Dopri 45 can be used. Lsodar is the fastest implicit integrator that was tested. Note that the simulation can easily be made faster by using a larger step size, at the cost of accuracy. Also, using larger step sizes will eventually lead to numerical instabilities. The user may therefore want to adjust the dynamics of the system, or set certain dynamics to steady state. It is thus important that models expose these parameters and allow easy configuration.

The achieved speed increase is considerable. However, this still requires about 18 hours for a one-year simulation. As this is longer than typical building energy simulations, we think that further research is desirable to reduce the simulation time further. 


\section{Conclusion}

We conclude that the analysis of algebraic loops, the optimization of Modelica code and the application of physical insight can lead to significant simulation time improvements. Analysis of the model time constants, avoiding system instabilities, using analytic Jacobians and proper integrator choice can also be important. These modifications were applied to a large building model where removal of all 'fast' dynamics allowed explicit integrators to perform well. Fixed step integrators can also be used if simulation results do not need to be very accurate. Euler integration performs very well in terms of computation time, allowing detailed office building simulations at a speed 500 times faster than real time.

Further work can focus on analysing and changing the problem structure in such a way that parallelization can be used efficiently. It should also be investigated up to which extent models can be made faster by changing the model dynamics, which allows larger time steps to be taken, without introducing too large errors. The proposed changes demonstrate that further symbolic processing in Dymola and OpenModelica is possible. We also propose to use analytic Jacobians by default for all Jacobian elements where an analytic Jacobian can be computed.

\section{Acknowledgements}

The authors acknowledge the financial support by the Agency for Innovation by Science and Technology in Flanders (IWT) for the PhD work of F. Jorissen (contract number 131012).

This research was supported by the Assistant Secretary for Energy Efficiency and Renewable Energy, Office of Building Technologies of the U.S. Department of Energy, under Contract No. DE-AC02-05CH11231.

This work emerged from the Annex 60 project, an international project conducted under the umbrella of the International Energy Agency (IEA) within the Energy in Buildings and Communities (EBC) Programme. Annex 60 will develop and demonstrate new generation computational tools for building and community energy systems based on Modelica, Functional Mockup Interface and BIM standards.

\section{References}

Ruben Baetens, Roel De Coninck, Filip Jorissen, Damien Picard, Lieve Helsen, and Dirk Saelens. Openideas - an open framework for integrated district energy simulations. In Building simulation 2015, submitted, Hyderabad, 2015.

François E. Cellier and Ernesto Kofman. Continuous System Simulation. Springer US, 2006.
Earl A. Coddington and Norman Levinson. Theory of ordinary differential equations. McGraw-Hill Book Company, Inc., New York-Toronto-London, 1955.

Dassault Systèmes. Dymola user manual, vol. 1, 2014.

Ernst Hairer and Gerhard Wanner. Solving Ordinary Differential Equations II: Stiff and Differential-Algebraic Problems. Springer-Verlag Berlin Heidelberg, 2002.

Alan C. Hindmarsh. Odepack, a systematized collection of ode solvers. IMACS transactions on scientific computation, 1:55-64, 1983.

Linda R. Petzold. Description of dassl: a differential/algebraic system solver. Technical report, Sandia National Labs., Livermore, CA (USA), 1982.

Linda R. Petzold. Automatic selection of methods for solving stiff and nonstiff systems of ordinary differential equations. SIAM journal on scientific and statistical computing, 4(1): 136-148, 1983.

Damien Picard and Lieve Helsen. Advanced Hybrid Model for Borefield Heat Exchanger Performance Evaluation, an Implementation in Modelica. In 10th International Modelica Conference 2014, pages 857-866, Lund, 2014.

Elijah Polak. Optimization, Algorithms and Consistent Approximations, volume 124 of Applied Mathematical Sciences. Springer Verlag, 1997.

Michael Tiller. Introduction to Physical Modeling with Modelica. Springer US, 2001.

Michael Wetter. Fan and pump model that has a unique solution for any pressure boundary condition and control signal. In Jean Jacques Roux and Monika Woloszyn, editors, Proc. of the 13-th IBPSA Conference, pages 3505-3512, 2013. URL http://simulationresearch.lbl.gov/ wetter/download/2013-IBPSA-Wetter.pdf.

Michael Wetter, Wangda Zuo, Thierry S. Nouidui, and Xiufeng Pang. Modelica buildings library. Journal of Building Performance Simulation, 7(4):253-270, 2014.

Michael Wetter, Marcus Fuchs, Pavel Grozman, Lieve Helsen, Filip Jorissen, Moritz Lauster, Dirk Müller, Christoph Nytsch-Geusen, Damien Picard, Per Sahlin, and Matthis Thorade. IEA EBC annex 60 modelica library - an international collaboration to develop a free open-source model library for buildings and community energy systems. In Building simulation 2015, submitted, Hyderabad, 2015.

Dirk Zimmer. Using Artificial States in Modeling Dynamic Systems : Turning Malpractice into Good Practice. In Proceedings of the 5th International Workshop on EquationBased Object-Oriented Modeling Languages and Tools, pages $77-85,2013$. 